Recibido: 1 junio 2016 Aceptado: 25 junio 2016 Publicado: 29 Julio 2016 Cita: Espinosa, G. (2016). «Dientes de sierra: una herramienta para el estudio de la estructura interactiva del discurso dialógico». Normas 6, 13-27. doi: $10.7203 /$ Normas.6.8828

\section{Dientes de sierra: una herramienta para el estudio de la estructura interactiva del discurso dialógico}

\author{
SAWTEETH: A TOOL TO STUDY THE \\ INTERACTIONAL STRUCTURE OF DIALOGIC \\ DISCOURSE
}

\author{
Guadalupe Espinosa Guerri \\ Universitat de València / Grupo Val.Es.Co
}

Resumen:

La presente investigación tiene como objetivo principal presentar las bases metodológicas para aplicar los dientes de sierra, una herramienta para el estudio de la interacción en los discursos dialógicos. Dicha herramienta se asienta sobre el sistema de unidades de la conversación coloquial de Val.Es.Co y aporta un esquema visual de la estructura interactiva de los géneros dialógicos. Para mostrar el funcionamiento de la herramienta, primero se describen las unidades del sistema Val.Es.Co sobre las que se aplican los dientes de sierra, seguidamente se presentan los símbolos que configuran la herramienta, las normas para combinar los símbolos y, finalmente, los criterios de análisis. El fin último de este artículo es mostrar una potente herramienta para el análisis de la estructura interactiva del discurso que puede ser utilizada con fines filológicos diversos, que aporta un punto de vista novedoso al Análisis de la Conversación y que abre múltiples vías de investigación.

PALABRAS CLAVE: dientes de sierra, herramienta de visualización, estructura interactiva del discurso dialógico, sistema de unidades de Val.Es.Co, Análisis de la Conversación.

Abstract:

The main objective of this paper is to present the methodological bases to apply Sawteeth, a tool to study the interaction in dialogical discourses. This tool is established on the Val.Es.Co unit's system of casual conversations and provides a visual schema of the interactional structure of dialogic genres. To show how it functions, we describe in the first place the units of the Val.Es.Co system that are used, next we present the symbols that form the tool, the rules to combine them and finally the analysis criteria. The last aim of this paper is to show a powerful tool for the analysis of the interactional structure of discourse that can be used with different philological goals, providing Conversational Analysis with a new point of view and opening many avenues for research.

KEYWORDS: Sawteeth, visualization tool, interactive structure of dialogical discourse, Val.Es.Co units system, Conversational Analysis. 
La herramienta denominada dientes de sierra (DS) es una herramienta de visualización creada para analizar y representar gráficamente la relación entre las intervenciones de un discurso dialógico y así obtener un esquema visual de su estructura interactiva como el que se puede apreciar en el siguiente ejemplo:

(1)

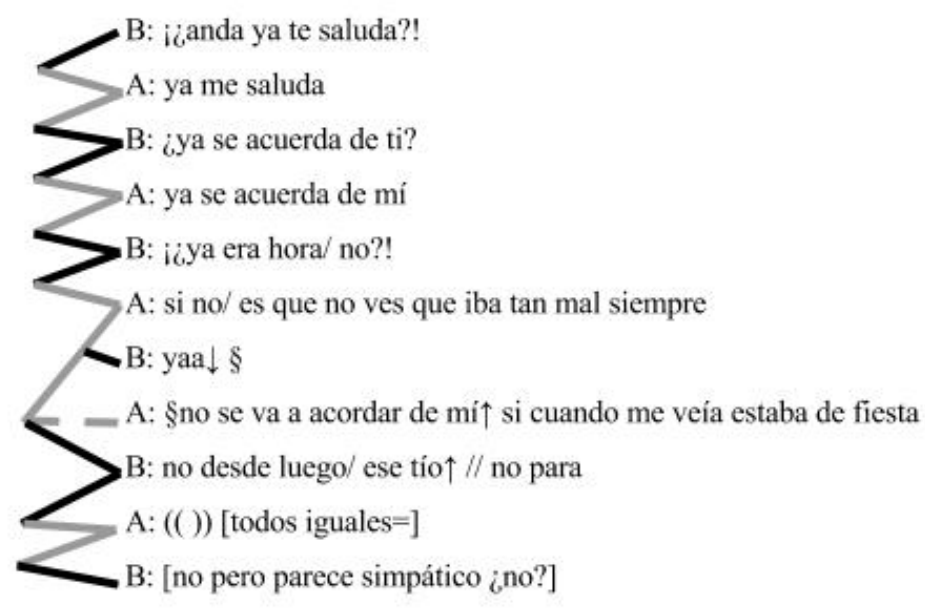

Al aplicar esta herramienta, el investigador ya no se enfrenta directamente al bloque de información homogénea que ofrece la transcripción, sino que dispone de un mecanismo que le permite estructurar toda esa información, establecer relaciones concretas y jerarquizar los elementos. En definitiva, se trata de un potente motor de análisis que se puede utilizar con distintos fines filológicos: detectar patrones interactivos, investigar el comportamiento dialógico de los hablantes o estudiar las características interactivas de géneros son algunas de las vías de investigación que abre esta herramienta.

Los DS surgieron en el seno del grupo Val.Es.Co. en 2013. Antonio Briz fue quien desarrolló dicha herramienta (2006, 2014 y en prensa) y posteriormente fue ampliada (Espinosa, en prensa) con un código de colores y algunos símbolos que completarían la herramienta. Fue creada especialmente para aplicarla sobre la propuesta de unidades de la conversación coloquial de este mismo grupo de investigación (Val.Es.Co, 2003, 2014) y así aportar un punto de vista diferente al estudio del coloquio. El presente artículo pretende, pues, dar a conocer esta herramienta y establecer una metodología de aplicación sistemática para que pueda ser utilizada por la comunidad científica de una forma homogénea y rigurosa. Es decir, el objetivo que motiva este estudio es un objetivo de naturaleza metodológica que se concreta en varios puntos: 1) describir brevemente las unidades discursivas sobre las que se aplican los DS, 2) presentar los símbolos que configuran esta herramienta y el código de colores con que se combina, 3) indicar cuáles son los pasos básicos que hay que seguir para empezar a utilizar la herramienta y 4) especificar las normas de representación de las unidades de análisis. Una vez hecho esto, se reflexionará sobre algunos aspectos relevantes de esta herramienta y finalmente se dedicará el último apartado a exponer las líneas de investigación en las que se está trabajando actualmente, así como las puertas que abre para estudios futuros.

\section{Metodología de APLICACIÓN DE LOS DS}

Se puede entender los DS como un lenguaje en sí mismo, es decir, como un sistema que posee un vocabulario y una sintaxis, porque presenta una serie de símbolos y esos símbolos se combinan siguiendo unas normas específicas. A continuación se ofrecerá toda la información necesaria para aplicar esta herramienta convenientemente. 


\subsection{Unidades sobre las que se aplica}

El sistema de Val.Es.Co divide el discurso en ocho unidades: subacto, acto, intervención, intercambio, turno, alternancia de turno, diálogo y discurso. De todas ellas, los DS se aplican sobre unidades del nivel macroestructural que se van a caracterizar a continuación aportando una definición operativa y los subtipos que presentan en algunos casos.

La intervención es laemisión de un hablante que reacciona a lo dicho por otro interlocutor y/o genera una reacción posterior (Val.Es.Co, 2014:17). El concepto de reacción, es clave, para la identificación de esta unidad: una reacción puede ser tanto verbal como no verbal y hace referencia a «cada una de las manifestaciones de la participación de un hablante en la conversación, aunque no constituyan por sí mismas un turno» (íbid., 17).

Hay distintos tipos de intervención definidas según la noción de reacción: a) las intervenciones iniciativas son aquellas que provocan una reacción, b) las reactivo-iniciativas son las que reaccionan a una intervención previa y generan otra intervención posterior, y las reactivas reaccionan a una intervención previa pero no provocan ninguna (íbid., 19). Además, se puede añadir un cuarto tipo de intervenciones, las independientes, que son aquellas que no reaccionan a ninguna intervención previa ni generan reacciones posteriores. Puesto que este tipo de intervenciones no ha sido descrito en ninguno de los trabajos anteriores del grupo, a continuación se incluye un ejemplo para entender mejor a qué hacemos referencia:

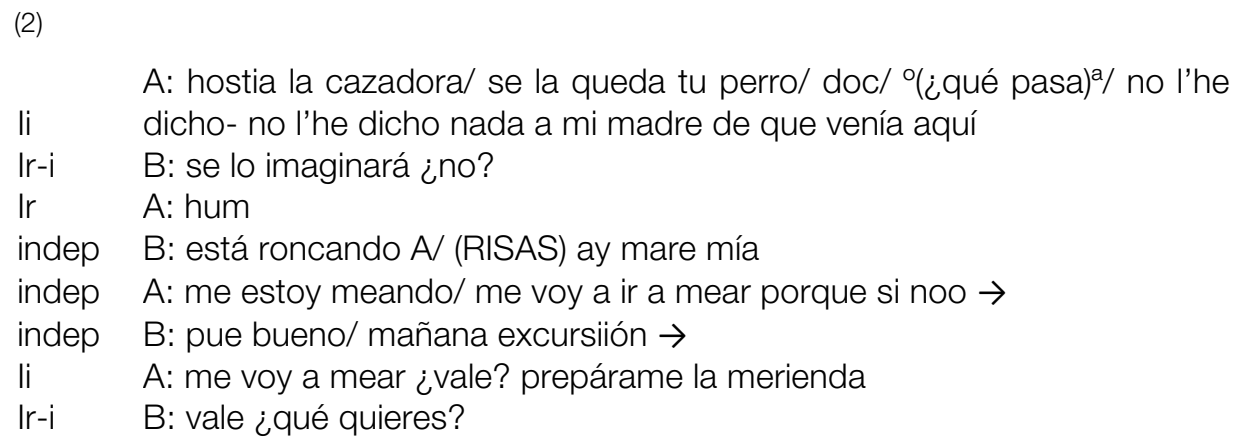

En este fragmento de transcripción hay tres intervenciones independientes sucesivas. Son independientes porque, a diferencia de las otras, no se relacionan de forma reactiva con ninguna intervención anterior ni posterior. B reacciona al hecho de que el perro está roncando y $A$ solo comenta la necesidad de ir al baño sin que esto genere la reacción de B. Luego B recuerda en voz alta la excursión del día siguiente, pero esto tampoco genera ninguna reacción explícita en A, quien insiste en que tiene que ir al baño hasta que, finalmente, consigue que B conteste.

Además, no todas la intervenciones independientes son iguales, sino que se pueden distinguir dos subtipos. Hay ocasiones en que la independiente se produce porque el hablante manifiesta verbalmente una sensación, un estado de ánimo o un sentimiento; es decir, reacciona a un estímulo interno, lo exterioriza pero no genera la reacción posterior de sus interlocutores. Es lo que sucede en el siguiente ejemplo:

(3)

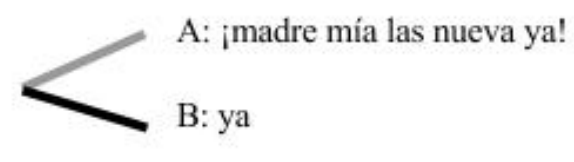

— A: estoy más cansá ((me tenía que quedar)) [aquí]

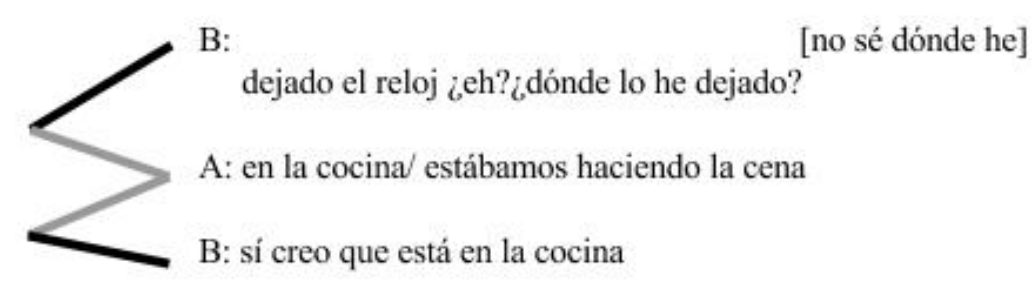


En este caso, intervención de A no reacciona a ningún estímulo externo, simplemente manifiesta su cansancio de forma explícita. Podríamos llamarlas, por tanto, independientes internas. En otros casos, en cambio, la independiente se produce porque algo del espacio donde se da la comunicación llama su atención. Hay que tener en cuenta que la conversación coloquial es un género que se suele dar en marcos interactivos cotidianos, (una casa, un bar, la calle, etc.) y a menudo, al mismo tiempo que se conversa también se puede ver la televisión o comer. Esto hace que de forma espontánea, los hablantes abandonen momentáneamente la conversación para atender a lo que pasa a su alrededor. Es lo que sucede en este ejemplo 4 cuando A reacciona al hecho de que B está hablando con la boca llena. Estas serían independientes externas:

(4)

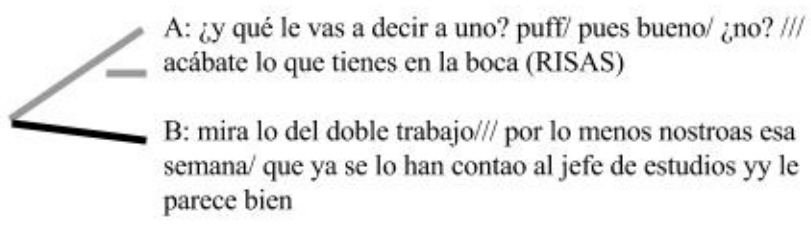

Las intervenciones suelen asociarse a cambios de hablante, pero esto solo se da cuando las intervenciones son continuas, es decir, que no sufren ningún tipo de interrupción. Sin embargo, a menudo se dan intervenciones discontinuas e intervenciones compuestas. Las primeras son intervenciones que han sufrido algún tipo de interrupción sin que el emisor deje de decir lo que se había propuesto (Val.Es.Co, 2014:20-21). Y las segundas se dan cuando un hablante emite dos intervenciones en un mismo turno, es decir, cuando en una misma intervención, una parte es reactiva de los dicho anteriormente y la otra es un nuevo inicio (íbid., 24-25). La sucesión de dos intervenciones constituye la unidad dialógica mínima, el intercambio.

Un turno (íbid., 23-31), por su parte, es una intervención que consigue aceptación social, esto es, el reconocimiento de los demás interlocutores. Esta distinción permite discernir un turno de intervenciones fáticas o de intervenciones que son ignoradas (voluntaria o involuntariamente) y que al no conseguir aceptación social no son turno. Además, esta diferencia entre turno e intervención es definitoria del sistema de unidades de Val.Es.Co frente a otras propuestas de segmentación del discurso. De forma paralela a lo que sucedía con la intervención y el intercambio, el turno también tiene su correspondiente unidad en el plano dialógico, la alternancia de turnos, que es la sucesión de, al menos, dos turnos.

El diálogo es un conjunto de intercambios sucesivos que empieza en una intervención iniciativaturno y termina en una intervención reactiva-turno. Y la unidad mayor del sistema, el discurso (Estellés y Pons, 2014:121-155), es aquella situada entre dos posiciones iniciales absolutas donde se produce una modificación de alguno de los parámetros de la conversación (el rol de los conversadores, la cantidad de conversadores envueltos en la conversación, la relación jerárquica entre ellos, el estatus social del intercambio o el modo espontáneo/planificado/semiplanificado).

Por tanto, los DS se aplican sobre las unidades intervención, intercambio, turno, alternancia de turno, diálogo y discurso; y quedan fuera del análisis las unidades acto y subacto.

\subsection{Símbolos y código de colores}

Los DS es una herramienta de visualización formada por seis símbolos y un código de colores que se aplican sobre las unidades descritas anteriormente mediante unas reglas de combinación concretas. Esos símbolos son los siguientes:

- Símbolo de intervención iniciativa:

- Símbolo de intervención reactiva

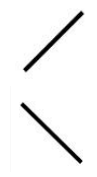

- Símbolo de intervención reactivo-iniciativa 
- Símbolo de intercambio

- Símbolo de intervención discontinua _ - -

- Símbolo de intervención independiente

- Símbolo de intervención no analizable

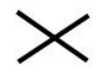

Sobre estos símbolos se aplica un código de colores para identificar qué hablante emite cada una de las intervenciones. Ese código variará en función del género dialógico al que se aplique y del número de hablantes. En la siguiente tabla se presentará el código para conversaciones coloquiales y entrevistas de 2 a 5 participantes:

\begin{tabular}{|c|c|c|}
\hline$N^{\circ}$ hablantes & $\begin{array}{l}\text { Conversación } \\
\text { coloquial }\end{array}$ & Entrevista $^{1}$ \\
\hline \multirow[t]{2}{*}{2} & A rosa & E. azul \\
\hline & $B$ verde & Edo. naranja \\
\hline \multirow[t]{3}{*}{3} & A rosa & E. azul \\
\hline & $B$ verde & Edo. naranja \\
\hline & C naranja & Edo1. rosa \\
\hline \multirow[t]{4}{*}{4} & A rosa & E. azul \\
\hline & B verde & Edo. naranja \\
\hline & C naranja & Edo1. naranja \\
\hline & D azul & Edo2. verde \\
\hline \multirow[t]{5}{*}{5} & $\overline{\text { A rosa }}$ & \\
\hline & B verde & \\
\hline & C naranja & \\
\hline & D azul & \\
\hline & E rojo & \\
\hline
\end{tabular}

Tabla 1: código de colores para conversaciones y entrevistas

Debido a las normas de estilo de la revista, en lugar de utilizar colores, se utiliza una escala de grises para representar las intervenciones de los distintos hablantes que aparezcan en los ejemplos. Concretamente, las intervenciones del hablante A serán grises y las de B, negras.

\subsection{Tres pasos básicos para utilizar los DS}

Los símbolos presentados en el apartado anterior se combinan en la práctica siguiendo tres sencillos pasos:

1) Cuestionarse ante cada cambio de hablante si genera alguna intervención posterior y cuál(es) exactamente:

(5)

$$
\begin{gathered}
\text { B: izanda ya te } \\
\text { saluda?! }
\end{gathered}
$$

A: ya me saluda

\footnotetext{
${ }^{1}$ E. es entrevistador y Edo., entrevistado. 
2) Unir las intervenciones que estén relacionadas según el criterio de inicio-reacción:

(6)

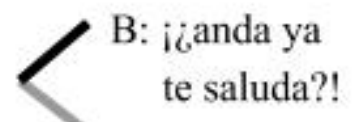

A: ya me saluda

3) Repetir la misma operación sucesivamente:

(7)

$$
\begin{aligned}
& \text { B: i¿anda ya te } \\
& \text { saluda?! } \\
& \text { A: ya me saluda } \\
& \text { B: ¿ya se acuerda } \\
& \text { de ti? }
\end{aligned}
$$

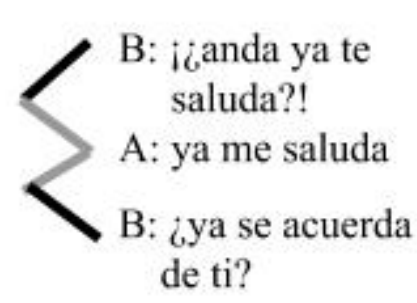

de ti?

Este es el funcionamiento más básico de la herramienta, el que se da cuando lo que hay en la conversación es una simple sucesión de intervenciones continuas. Pero con esta herramienta también se pueden visualizar los otros tipos de intervenciones y las unidades superiores. En el siguiente apartado se detalla el modo de representarlas.

\subsection{Representación de las unidades de análisis}

A continuación se presenta de forma exhaustiva el modo de representar las intervenciones discontinuas, continuas, independientes y no analizables, así como los diálogos y los discursos.

Las intervenciones discontinuas son intervenciones que han sido interrumpidas con reacciones de tipo fático o colaborativo, pero que mantienen el turno. Para representar este fenómeno, se ha optado por el siguiente método:

(8)

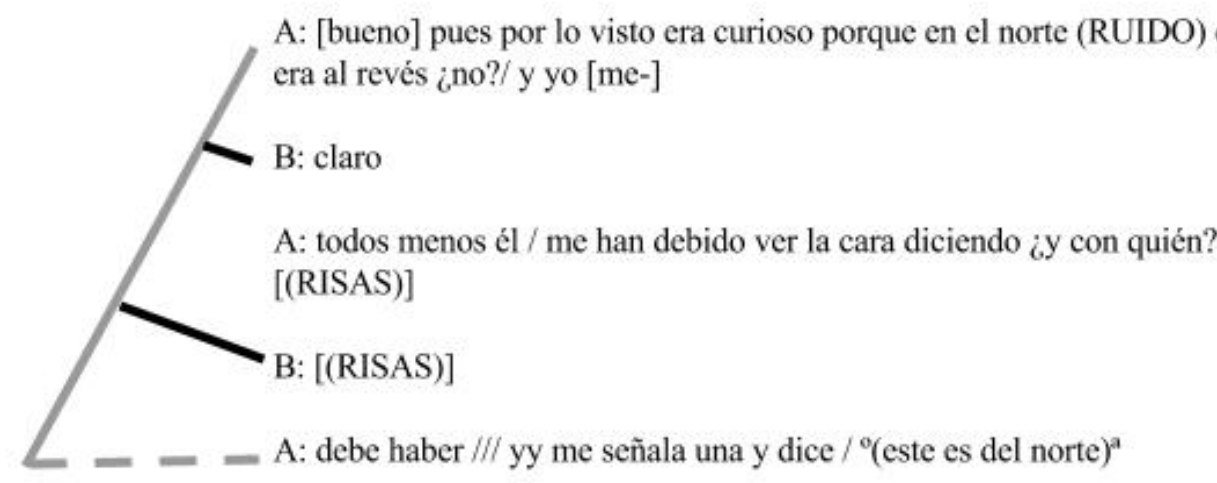

La intervención discontinua se marca con una barra de inicio y con líneas discontinuas en el último cambio de hablante del emisor responsable de dicha intervención, en este caso, A. Y las intervenciones fáticas de B que van interrumpiendo la intervención principal sin robarle el turno se marcan con barras reactivas.

También se pueden encontrar intervenciones discontinuas que son solo reactivas. En estos casos, el esquema gráfico se invierte, es decir se marca el primer cambio de hablante de la discontinua con las líneas discontinuas y luego se dibuja un barra reactiva: 
(9)

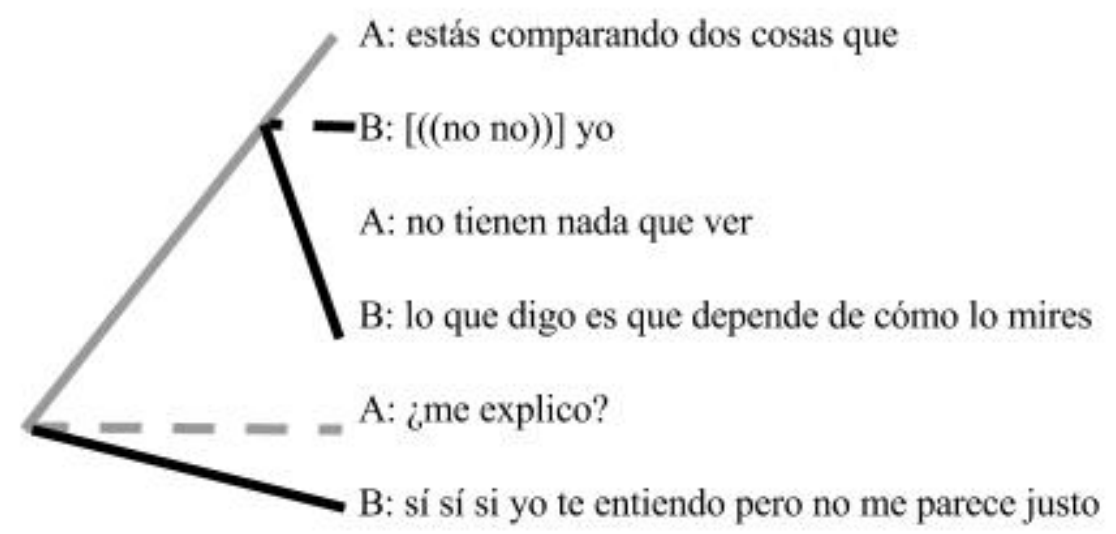

En este ejemplo se puede observar una intervención discontinua de A que es iniciativa, y dentro de esta, otra discontinua de $\mathrm{B}$ que es solo reactiva.

Además, puede suceder que una intervención no reaccione a nada anterior ni genere ninguna intervención posterior, en ese caso se utilizará el símbolo de intervención independiente (ver apartado 2.2), de modo que esa intervención no quedará unida a ninguna otra, marcando así su carácter autónomo:

(10)

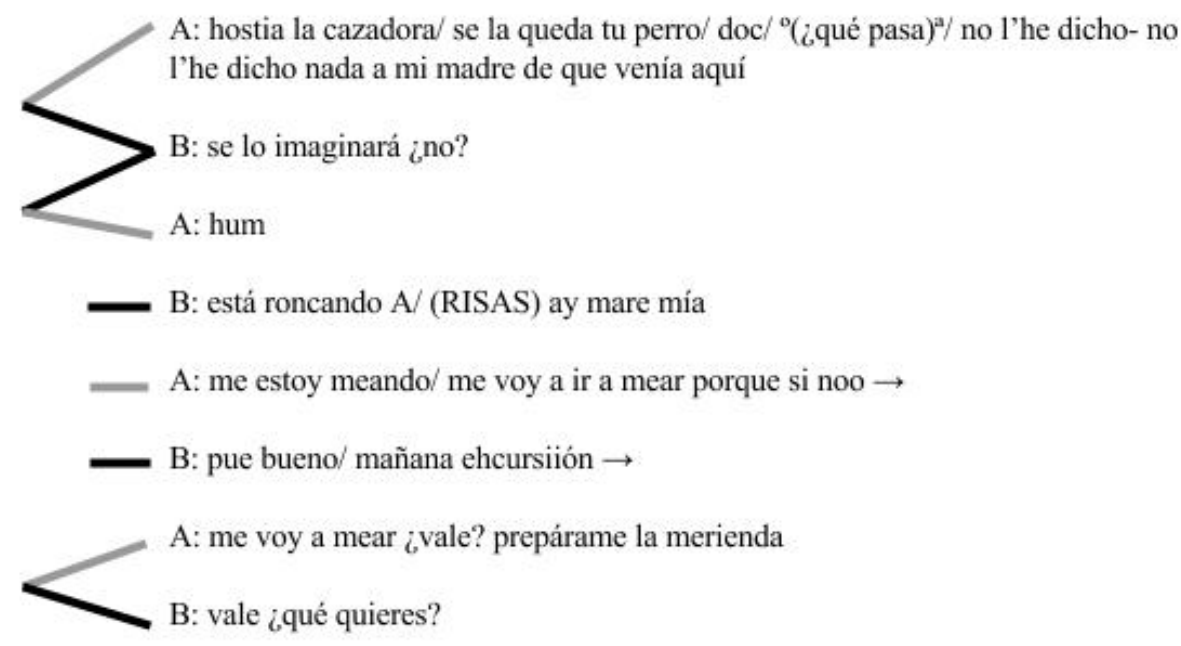

Asimismo, hay intervenciones no analizables, bien porque el contenido semántico no se ha podido transcribir o bien porque, aunque el contenido se ha transcrito, el analista no tiene los datos suficientes como para determinar a qué intervención concreta está reaccionando ni qué intervenciones posteriores genera. El símbolo escogido, tal y como se ha presentado en el apartado 2.2 es una cruz. En el siguiente fragmento, la intervención del hablante desconocido no se puede interpretar porque no tiene contenido semántico:

(11)

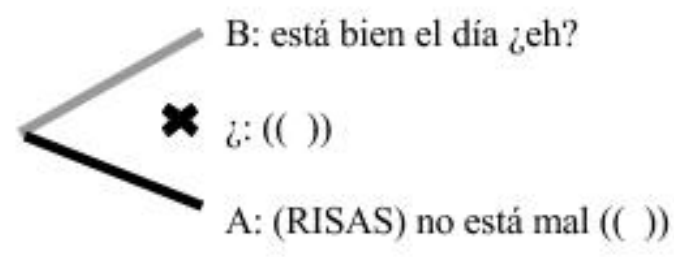


En cuanto a las unidades superiores del modelo, los diálogos y discursos, su representación es la siguiente: los diálogos se generan con esta herramienta cuando hay una intervención reactiva que no se puede unir con ninguna intervención posterior, ya que en el sistema de unidades de Val.Es.Co (2014), un diálogo viene limitado por una intervención-turno iniciativa al inicio y por una intervenciónturno reactiva al final. Se dejará, pues, un espacio en blanco como en el siguiente ejemplo:

(12)

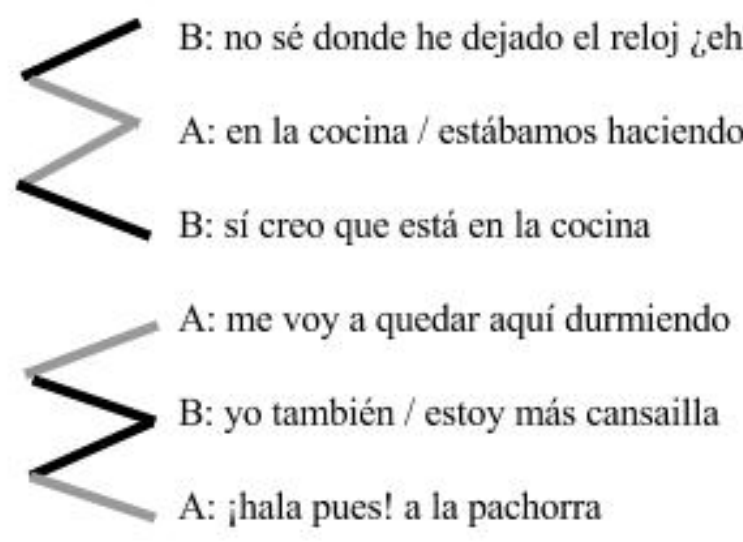

Y finalmente, para marcar el inicio y el fin de la unidad mayor, el discurso, se utiliza una barra horizontal como se puede apreciar en este ejemplo:

(13)

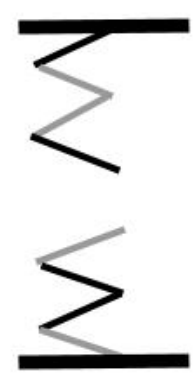

Estos son, pues, todos los elementos necesarios para poder representar las unidades que configuran la estructura de la conversación coloquial. Solo queda establecer los criterios para poder llevar a cabo el análisis visual de forma homogénea, de modo que, en el apartado siguiente se ofrecerán algunos factores que pueden guiar al usuario a la hora de analizar y visualizar la estructura de la conversación coloquial.

\subsection{Criterios para el análisis de la estructura interactiva}

La aplicación de los DS se basa en un análisis previo de las intervenciones. En ese análisis el investigador se plantea, como se ha mencionado anteriormente, dos cuestiones fundamentales: a qué reaccionan los hablantes y qué reacciones generan sus intervenciones; lo cual supone, a su vez, determinar qué tipo de intervenciones se dan en el transcurso de la conversación. Para llevar a cabo ese análisis se tienen en cuenta diversos factores:

a) El tema: una ruptura temática es un factor que opera en muchos casos para determinar cuándo hay final de diálogo e inicio de otro. 
(14)
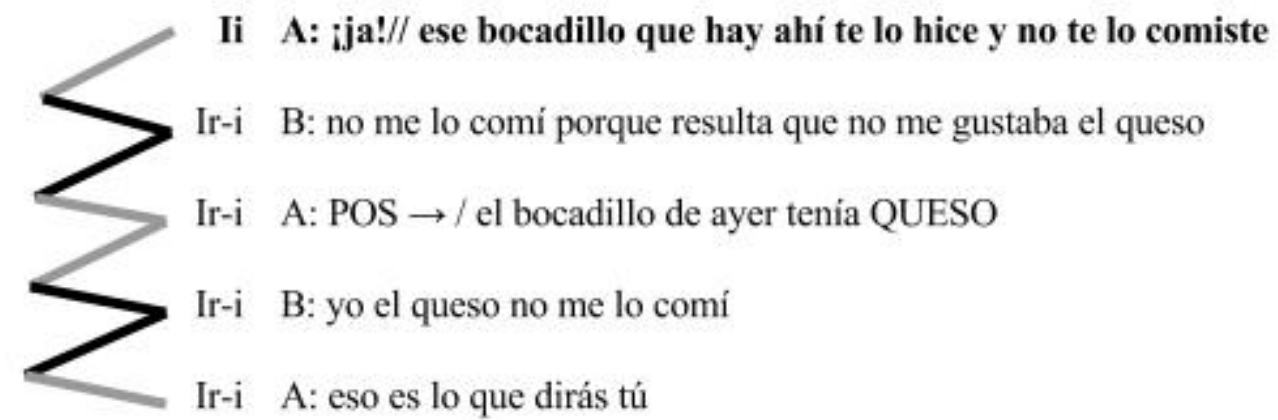

Ir-i B: no me lo comí porque resulta que no me gustaba el queso

Ir-i A: POS $\rightarrow /$ el bocadillo de ayer tenía QUESO

Ir-i B: yo el queso no me lo comí

Ir-i A: eso es lo que dirás tú

x B: aah

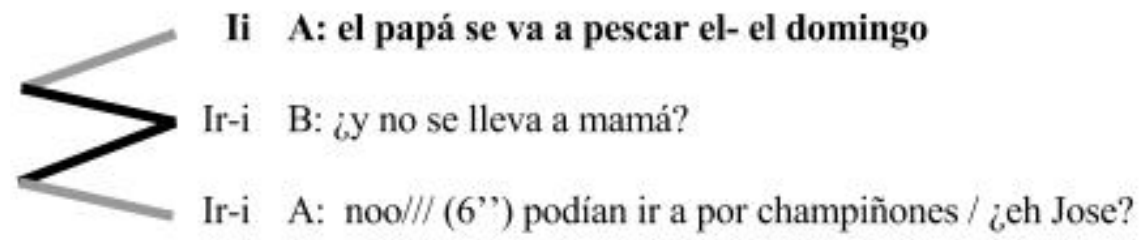

En este fragmento se puede dividir en dos bloques temáticos: el primero lo ocupan las cinco primeras intervenciones, en las que A y B discuten sobre el bocadillo. Guardan una coherencia temática que nos permite etiquetar la primera como iniciativa y el resto como reactivo-iniciativas que van añadiendo información al tema. Entre el primer y segundo bloque temático hay una intervención marcada con una cruz que no podemos etiquetar porque no tenemos información suficiente como para saber a qué está reaccionando B y en cuál de los dos bloques se inserta, podría ser incluso una reacción a algo que se ha caído. Las tres intervenciones siguientes comparten un tema totalmente distinto a las del primer bloque, puesto que se pasa a hablar de la excursión del padre. La coherencia temática es lo que permite catalogar la primera intervención de A como iniciativa de un nuevo diálogo y el resto como reactivo-iniciativas.

Sin embargo, aunque el tema es un factor determinante en muchos casos como el que se acaba de exponer, hay ocasiones en las que el cambio de tema no garantiza que haya un inicio de diálogo, como sucede en el siguiente ejemplo: 
(15)

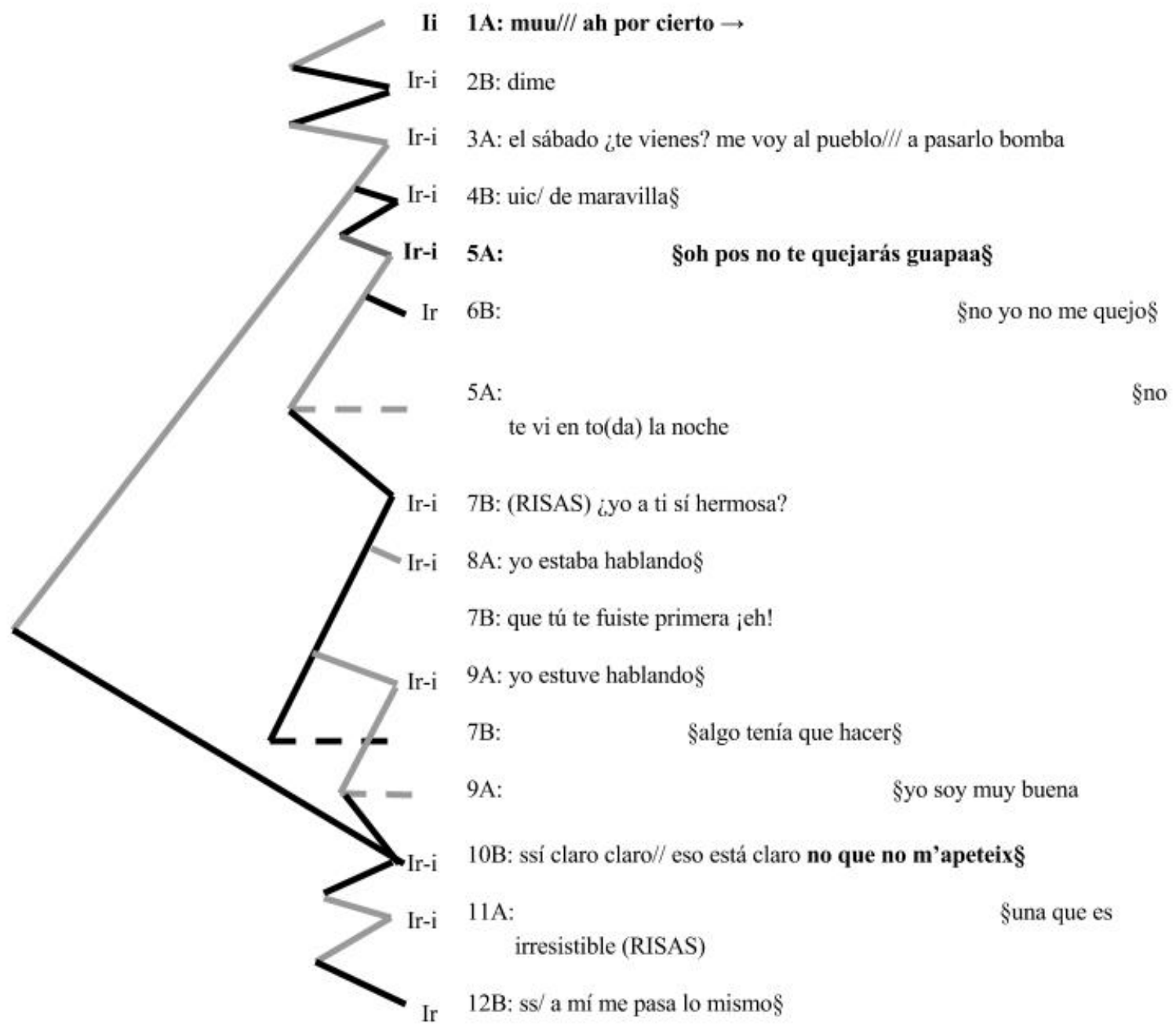

En este fragmento hay dos bloques temáticos, el primero trata sobre la idea de ir al pueblo el sábado y va desde la intervención $1 \mathrm{~A}$ hasta $6 \mathrm{~B}$; mientras que en el segundo se habla sobre lo ocurrido en la discoteca, entre las intervenciones $5 \mathrm{~A}$ y $12 \mathrm{~B}$. Sin embargo, el cambio de tema no genera inicio de diálogo, sino que los dos temas están entrelazados, y en mitad del segundo bloque, la intervención 10B contesta a la pregunta inicial de A recuperando el primer tema del que se habían desviado. Esto es así porque, aunque el tema es un criterio a tener en cuenta para la clasificación de las intervenciones, también se tienen en cuenta otros factores de índole más estructural, como los que se van a exponer a continuación.

b) Mantenimiento de la voz: cuando un hablante mantiene la voz dominante a pesar de haber cambio de hablante, este suele ser un buen indicio de que el la intervención es discontinua y de que el hablante no pierde el turno aunque el otro le interrumpa. Nos referimos a casos como el siguiente: 
(16)

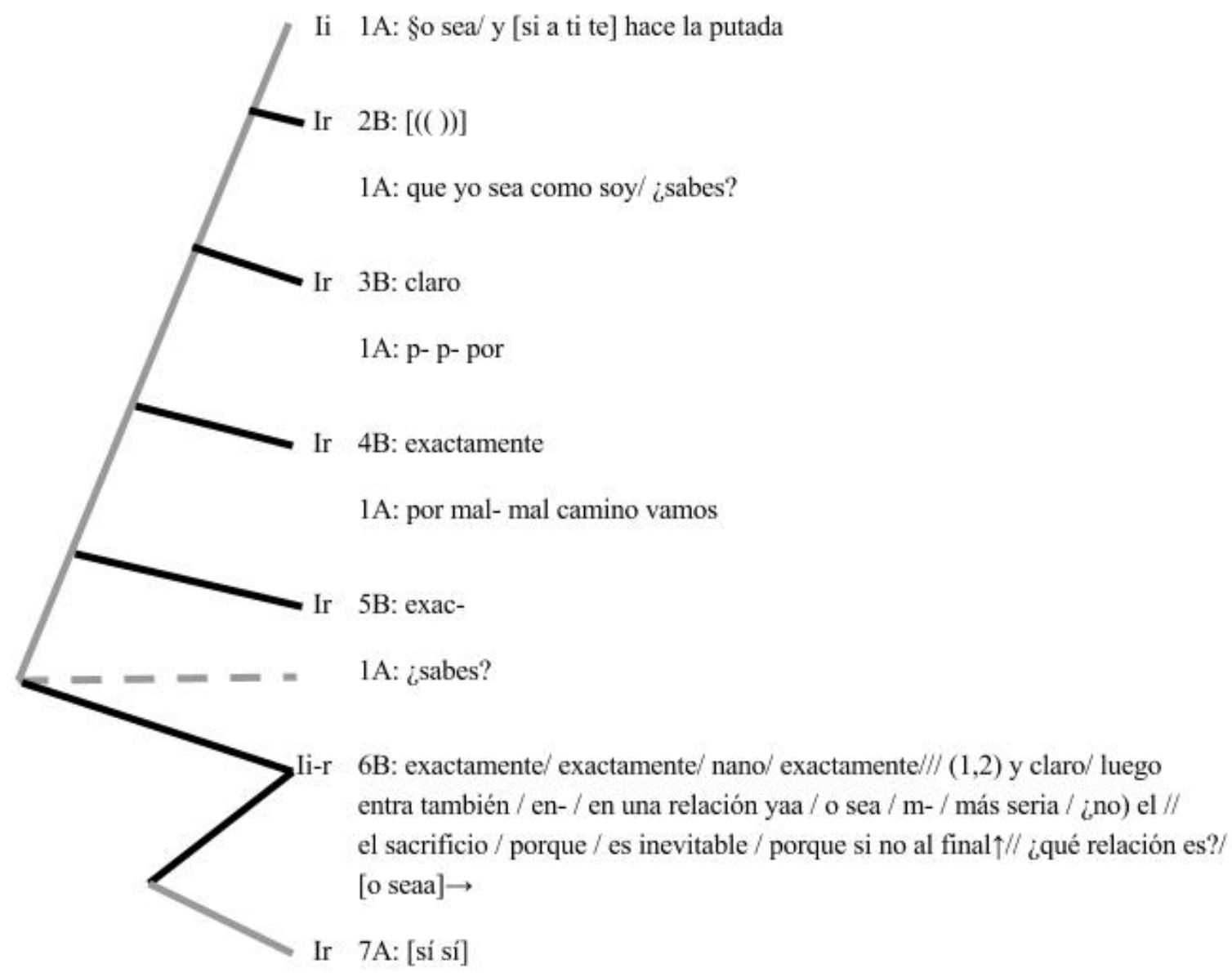

Lo que sucede aquí es que A está contándole a B los problemas que tiene con su novia, y en este fragmento lo hace mediante una intervención discontinua. La intervención 1A es interrumpida varias veces por las intervenciones reactivas de $\mathrm{B}(2 \mathrm{~B}, 3 \mathrm{~B}, 4 \mathrm{~B}$ y $5 \mathrm{~B})$ que son de tipo colaborativo pero no llegan a robarle el turno hasta la intervención $6 \mathrm{~B}$, la cual sí que consigue la reacción posterior de $7 \mathrm{~A}$.

c) Repeticiones: en muchas ocasiones, las repeticiones de palabras o grupos de palabras son indicio de intervenciones reactivo-iniciativas.

(17)

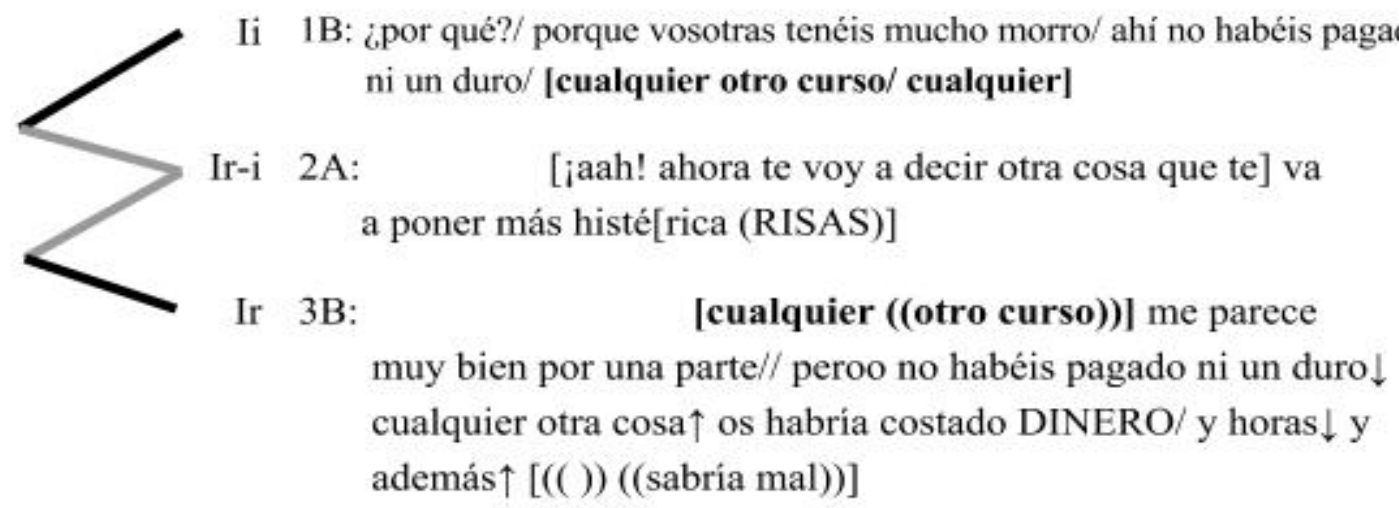

En este fragmento, las intervenciones $1 \mathrm{~B}$ y $3 \mathrm{~B}$ se podrían confundir con una intervención discontinua, ya que $B$ no parece reaccionar a lo que dice $A$. En cambio, el hecho de que en 3B se 
vuelva a repetir "cualquier otro curso" indica que A le ha robado la voz y, por tanto, B necesita recuperar lo dicho anteriormente para seguir con su argumentación.

d) Deícticos: los elementos deícticos tienen la misma función que las repeticiones, es decir, suelen ser indicios de intervenciones reactivas.

(18)
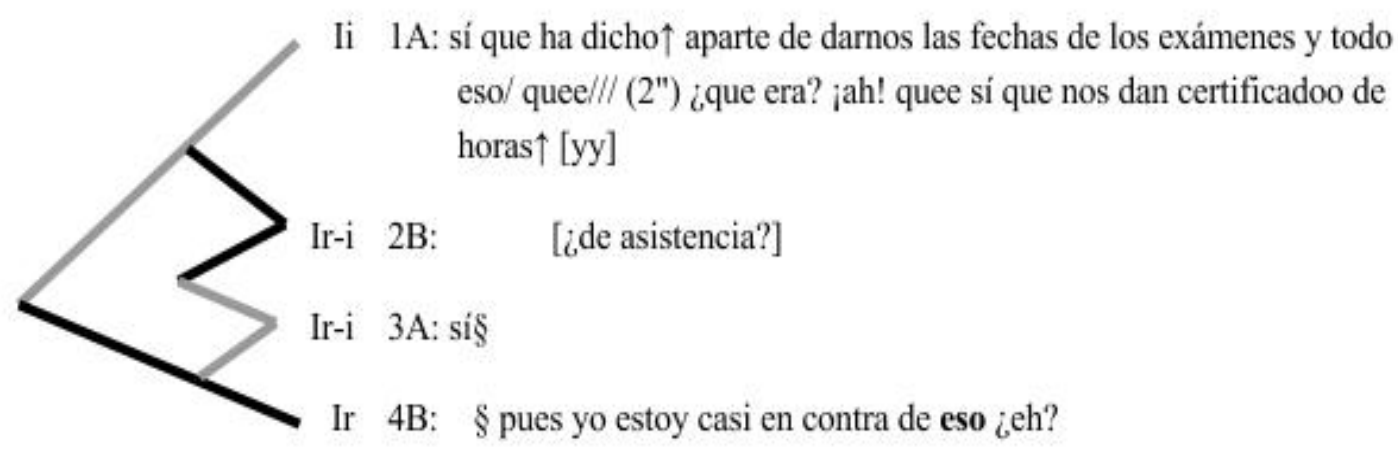

El deíctico "eso" indica que 4B es una intervención reactiva. Por una parte reacciona a 1A porque "eso" hace referencia al hecho de que les den el certificado, pero también reacciona a 3A porque solo cuando A le confirma que sí se trata del certificado de asistencia, B puede emitir su juicio al respecto. Se trata, por tanto, de una intervención reactiva con dos inicios, y el elemento que permite este análisis es, precisamente, el deíctico.

e) Partículas discursivas: muchas partículas discursivas, según la posición en la que se encuentres, pueden ayudar a clasificar las intervenciones en un tipo y otro. Por ejemplo, la partícula por cierto, al inicio de intervención suele indicar con bastante frecuencia que la intervención es iniciativa y de inicio de diálogo.

(19)

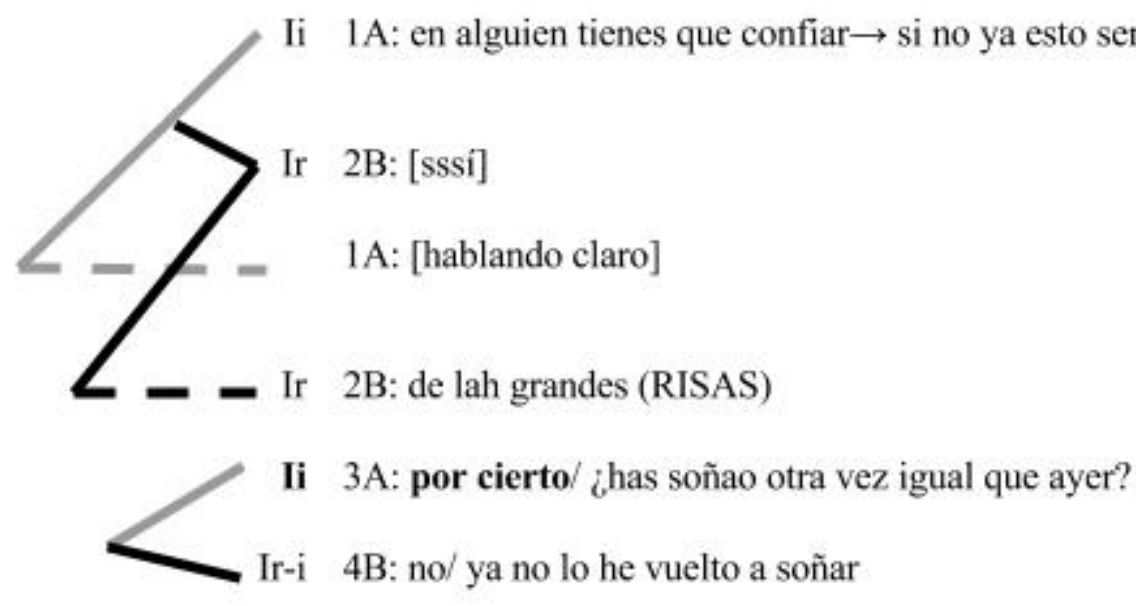

Este es un ejemplo claro de cuando la partícula por cierto indica la existencia de una intervención iniciativa. Llegados a este punto, el lector habrá percibido que los factores que aquí se están presentando como criterios para clasificar las intervenciones no actúan en solitario, sino que a menudo intervienen varios factores. En el caso que se acaba de presentar, la partícula discursiva es uno de los factores, pero el cambio brusco de tema también ayuda a realizar dicho análisis.

e) Elementos prosódicos: elementos como los solapamientos, las pausas, los tonemas, etc., también son valiosos recursos a tener en cuenta a la hora de catalogar las intervenciones. En el caso anterior, por ejemplo, el solapamiento que se produce en las intervenciones 2B y $1 \mathrm{~A}$ es lo que permite etiquetarlas como intervenciones discontinuas. Y además, cuando el análisis es dudoso, la 
posibilidad de acceder a la grabación original y a la entonación de las intervenciones suele aportar soluciones claras.

f) Longitud de las intervenciones: cuanto más larga es una intervención, más probabilidades hay de que los interlocutores se vean obligados a reaccionar a ella. En cambio, las intervenciones cortas pueden pasar más desapercibidas y, por tanto, pueden ser ignoradas con más facilidad.

Además de estos criterios necesarios para aplicar correctamente los DS, conviene tener en cuenta algunos aspectos relevantes sobre esta herramienta con el fin entender cuál es su función dentro del Análisis de la Conversación y qué puede aportar de nuevo a esta disciplina.

\section{ALGUNAS CONSIDERACIONES}

En primer lugar, los DS es una herramienta de visualización categórica, ya que los datos con los que se trabajan no son numéricos, sino que se trata de unidades de la conversación coloquial, es decir, elementos lingüísticos. Además, es analógica, porque su aplicación es manual. No se dispone todavía de un programa informático con el que digitalizar la herramienta, aunque esta es una de las líneas de investigación en las que se podrá trabajar en el futuro y que, sin duda, ayudará al desarrollo y expansión de la herramienta. Asimismo, también se ha contemplado la posibilidad de que pueda ser utilizada en el programa de transcripción Elan, cuyos archivos tienen una orientación horizontal. Por ello, los DS también permiten una visualización apaisada, aunque todavía no se ha trabajado en esta línea.

Esta herramienta se podrá utilizar en cualquier corpus:

- Que esté transcritos en papel, aunque la fuente puede ser tanto oral como escrita.

- Que sea un corpus de géneros dialógicos (conversaciones, entrevistas, debates, etc.) ya que es una herramienta diseñada para el estudio de la interacción. No es apta para el estudio de géneros monológicos.

- Que esté dividido en cambios de hablante, o que el investigador lo divida previamente de forma manual.

- Que incluya el máximo de información posible sobre aspectos como las pausas, solapamientos, tonemas, etc., ya que, aunque no es imprescindible, ayuda al investigar a decidir a qué intervención reacciona un hablante, qué tipo de intervención se trata o si hay inicio de diálogo, entre otras cosas.

Hay que destacar que en la utilización de los DS, interviene una parte interpretativa y otra sistemática. Interpretativa porque el analista debe buscar las intervenciones que están relacionadas entre sí, tal y como se ha explicado anteriormente. Es decir, la decisión de qué reacciona a qué corresponde al investigador, aunque, obviamente, esto se realiza teniendo en cuenta una serie de criterios (ver apartado 2.5). Pero esta herramienta también es sistemática porque al unir gráficamente las intervenciones que están relacionadas, los patrones van apareciendo por sí mismos. Es decir, el esquema gráfico que se genera nace de la utilización de unos símbolos establecidos y la combinación de esos símbolos en función de la relación entre intervenciones iniciativas y reactivas. Esto quiere decir que, dos analistas que conozcan el funcionamiento de la herramienta y tengan en cuenta los mismos criterios deberán obtener los mismos patrones. Se puede discrepar a la hora de determinar a qué intervención concreta reacciona un hablante, pero este es un problema del análisis.Si se coincide en el análisis, el patrón obtenido será siempre el mismo.

El investigador debe saber que el esquema que resulta de la utilización de los DS sobre un diálogo no es lo mismo que se puede observar a simple vista sin la herramienta. No se trata de una traducción literal de la transcripción con un "dibujo bonito", sino que lo que se obtiene es una esquema gráfico de la estructura interactiva mediante la cual se pueden ejecutar los distintos movimientos conversacionales. Es decir, lo que se ve con los DS es un esquema de las distintas estructuras de que disponen los hablantes para llevar a cabo una secuencia de preguntas y respuestas, un relato conversacional, una secuencia lateral, una secuencia de inserción, etc. No se trata de ver los fenómenos conversacionales desde un punto de vista gráfico, sino de ver la estructura que subyace a esos fenómenos descritos por otros autores bajo criterios sobre todo temáticos. Para aplicar los DS también se tiene en cuenta el criterio temático porque es uno de los elementos que sirven para determinar cuándo una intervención es iniciativa, reactivo-iniciativa o reactiva, sin embargo, este criterio se combina con otros de índole más formal, como la aparición de marcadores discursivos, repeticiones, solapamientos, etc. Al no ser el tema el criterio principal, 
sino uno más entre varios, esto hace que los patrones obtenidos por los DS sean de índole más estructural. Se podría decir, a modo de resumen, que los DS ofrecen una radiografía del esqueleto de la conversación.

\section{LÍNEAS DE INVESTIGACIÓN PRESENTES Y FUTURAS}

La herramienta expuesta en este artículo constituye una herramienta idónea para el estudio de la macroestructura de los discursos dialógicos, más concretamente, de la relación que se establece entre las intervenciones de los hablantes según el criterio básico de inicio y reacción de Val.Es.Co. Partiendo de esta consideración, los DS abren múltiples vías de investigación, algunas de las cuales todavía están por explorar y otras en las que ya se está trabajando:

a) Detectar patrones interactivos recurrentes: el esquema gráfico que se obtiene al aplicar la herramienta ha facilitado la detección de patrones interactivos recurrentes. Con ellos se ha podido elaborar una tipología de patrones interactivos, es decir, de tipos de movimientos conversacionales que se repiten constantemente en la interacción. Además, una vez establecida esa tipología, el propio inventario de patrones servirá para detectar fácilmente todo aquello que no encaje dentro de los patrones ya establecidos, y que probablemente indique la existencia de un nuevo patrón o de una anomalía interesante para ser estudiada más en profundidad (Espinosa y García-Ramón, en preparación).

b) Comparación intergenérica: los DS ofrecen un esquema gráfico fácil de comparar que permite ver las diferencias y semejanzas estructurales entre la conversación coloquial, la entrevista, el debate, la tertulia, etc. Ahora mismo se está trabajando en la comparación de la entrevista (García-Ramón, en preparación) y la conversación coloquial (Espinosa, en preparación).

c) Comparación interlingüística: del mismo modo que se puede utilizar la herramienta para comparar la estructura interactiva de distintos géneros, también se puede emplear para detectar posibles diferencias entre la forma de conversar en distintas lenguas.

d) Español como lengua extranjera: esta es una herramienta óptima para la enseñanza a estudiantes extranjeros de las estrategias conversacionales que se utilizan en el español, ya que, posiblemente, sean diferentes a las de su cultura de origen.

Tras lo dicho a lo largo de estas páginas, se espera haber aportado toda la información metodológica suficiente para la utilización de los DS, ya que se trata de una herramienta que puede arrojar nuevas ideas al estudio de la estructura interactiva de la conversación coloquial o de cualquier otro género dialógico.

\section{ABREVIATURAS}

AC: Análisis de la Conversación

DS: Dientes de sierra

\section{BIBLIOGRAFÍA}

Briz Gómez, Antonio (2006): «La segmentación de una conversación en diálogos», en Oralia, 9, 45-71 [en línea]: $<$ http://www.valesco.es/web/Val.Es.Co/Miem bros/Antonio\%20Briz/Antonio\%20Briz/Oralia \%202006/Briz\%20(segundas).pdf> [consultado el 30/09/2015].

Briz Gómez, Antonio (en prensa): El relato coloquial, presentado en el 280 Congreso de his- panistas italianos, Pisa (27-30/11/2013).

Briz Gómez, Antonio (2014): Apéndice 1. Definición, relaciones entre unidades y modos de visualizarlas, Valencia, Universitat de València (inédito).

Briz Gómez, Antonio y Grupo Val.Es.Co. (2003): «Un sistema de unidades para el estudio del lenguaje coloquial», en Oralia, 6, pp. 7-61.

Grupo Val.Es.Co (2014): «Las unidades del discurso oral. La propuesta Val.Es.Co. de segmentación de la conversación (coloquial) " en Cortés, L. (coord.), Estudios de Lingüística del Español 35, pp. 13-73 [en línea]: <http://infoling.org/elies/35/elies35.1.pdf> [consulta: 03/12/2014]. 
Espinosa Guerri, Guadalupe (en prensa): Hacia una visualización sistemática de la conversación coloquial.

Espinosa Guerri, Guadalupe (en preparación): Aportaciones al estudio de la estructura interactiva de la conversación coloquial española mediante una herramienta de visualización.

Espinosa Guerri, Guadalupe y Amparo, García-Ramón (en preparación) : Applying visualization tools to analyze turn-taking systems in conversations and interviews.

Estellés Arguedas, María. y Salvador Pons Bordería (ed.) (2014): «Absulute initial position», en Discourse Segmentation in Romance Languages, Grupo Val.Es.Co/ IUIMA/Universitat de València, Jhon Benjamins, P\&BNs, vol.250, (pp. 121-155).

García-Ramón, Amparo (en preparación): Aportaciones al estudio de la estructura interactiva de la entrevista mediante una herramienta de visualización. 
Dientes de sierra: una herramienta para el estudio de la estructura interactiva del discurso dialógico | Guadalupe Espinosa 